\title{
Pion-induced Drell-Yan processes and the flavor-dependent EMC effect
}

\author{
D. Dutta, ${ }^{1}$ J. C. Peng, ${ }^{2}$ I. C. Cloët ${ }^{3}$ and D. Gaskell ${ }^{4}$ \\ ${ }^{1}$ Department of Physics, Mississippi State University, Mississippi State, Mississippi 39762, USA \\ ${ }^{2}$ Department of Physics, University of Illinois at Urbana-Champaign, Urbana, Illinois 61801, USA \\ ${ }^{3}$ Department of Physics, University of Washington, Seattle, Washington 98195, USA \\ ${ }^{4}$ Thomas Jefferson National Accelerator Facility, Newport News, Virginia 23608, USA
}

\begin{abstract}
Pion-induced Drell-Yan processes are proposed as a potential tool to measure the flavor dependence of the EMC effect, that is, the flavor-dependent modification of quark distributions in the nuclear medium. Existing pionic Drell-Yan data are compared with calculations using a recent model for nuclear quark distributions that incorporates flavor-dependent nuclear effects. While no firm conclusions can yet be drawn, we demonstrate that existing Drell-Yan data seem to imply a flavor dependence of the EMC effect. We highlight how pion-induced Drell-Yan experiments on nuclear targets can access important new aspects of the EMC effect, not probed in deep inelastic scattering, and will therefore provide very stringent constrains for models of nuclear quark distributions. Predictions for possible future pion-induced Drell-Yan experiments are also presented.
\end{abstract}

PACS numbers: 13.85.Qk, 14.20.Dh, 21.65.Cd, 24.85.+p

The depletion of the nuclear structure functions in the valence quark region was discovered in 1983 by the European Muon Collaboration (EMC) in a muon-induced deep inelastic scattering (DIS) experiment [1], and is now known as the EMC effect. The EMC effect provides clear evidence that the quark distributions in nuclei are modified compared to those of free nucleons. The observation of the EMC effect was unexpected, since the nuclear binding energy is orders of magnitude smaller than the energy scale probed in DIS. Other DIS experiments using electron [2], muon [1, 3] and neutrino [4] beams have now confirmed the EMC effect over a broad range of nuclear masses $(A)$ and momentum transfers $\left(Q^{2}\right)$. The improved precision of these experiments, including the recent experiments on light nuclei [5, [6], have provided a detailed and multi-dimensional view of the nuclear modification of the quark distributions. The EMC effect has also been experimentally verified in the time-like region using both the pion- and proton-induced Drell-Yan processes 7 [9].

Despite a quarter century of significant experimental and theoretical effort, the specific origins of the observed $A$ dependence of the nuclear quark distributions have yet to be unambiguously identified. Attempts to explain the EMC effect have led to a large collection of theoretical models 10, 11], many of which are capable of describing the essential features of the data, however the underlying physics mechanisms in each model are often very different. Therefore, it appears likely that before the true origins of the EMC effect are understood new experiments are necessary, which explore aspects of the EMC effect not effectively probed in DIS, and will therefore help distinguish between the various models. In this Letter we explore the effectiveness of pion-induced Drell-Yan processes as a tool to investigate new aspects of the EMC effect [12, 13], in particular its flavor dependence.

A new calculation of the modifications of nucleon quark distributions in the nuclear medium has recently been reported [14, 15]. In this approach, by Cloët, Bentz and Thomas (CBT), the Nambu-Jona-Lasinio model is used to describe the coupling of the quarks in the bound nucleons to the scalar and vector mean fields inside a nucleus. These nucleon quark distributions are then convoluted with a nucleon momentum distribution in the nucleus to generate the nuclear quark distributions [14]. An important feature of this model is that for $N \neq Z$ nuclei (where $N$ and $Z$ refer to the number of neutrons and protons) the isovector-vector mean field (usually denoted by $\rho^{0}$ ) will affect the up quarks differently from the down quarks in the bound nucleons. Therefore, this model has a novel prediction that the $u$ and $d$ quarks have distinct nuclear modifications for $N \neq Z$ nuclei. In other words, the EMC effect is flavor sensitive and depends on the $N / Z$ ratio of the nucleus 14. This flavor-dependent EMC effect was also shown [15, 16] to account for a large fraction of the discrepancy between the $\mathrm{NuTeV}$ collaboration measurement 17] of the weak mixing angle and the world average.

Figure 11 shows the prediction for the nuclear dependence of quark distributions in the CBT model. The solid curve is the result for the usual EMC effect in symmetric $(N=Z)$ nuclear matter, that is, the ratio $F_{2}^{A} / F_{2}^{D}$, where $F_{2}^{A}$ and $F_{2}^{D}$ are the per-nucleon structure functions of the nucleus and the deuteron, respectively. ${ }^{1}$ The calculation is in good agreement with the data extracted by Sick and Day [18]. For an isoscalar nucleus the $u$ and $d$ quark distributions are modified in the same manner, however for a $N \neq Z$ nucleus the $u$ and $d$ quark distributions are predicted to undergo different modifications in the CBT model. For example, the dashed and dot-dashed curves in Fig. 1 are, respectively, the predicted ratio of the $u$ and $d$-quark distributions in a gold nucleus to those in

\footnotetext{
1 The CBT results used throughout this paper are calculations performed for asymmetric nuclear matter. Results for particular nuclei are obtained by choosing the $N / Z$ ratio equal to the nucleus in question. The deuteron results are obtained from a combination of free proton and neutron quark distributions.
} 


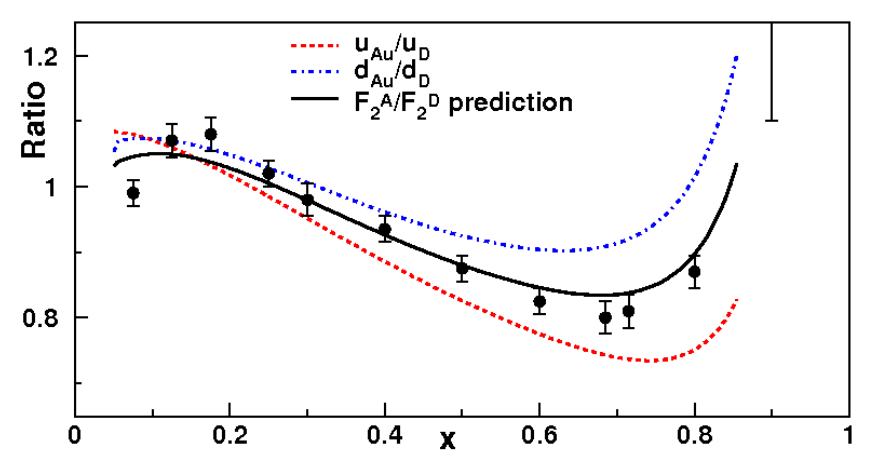

FIG. 1: Ratios of quark distributions and structure functions in nuclear matter versus the deuteron plotted as a function of Bjorken- $x$, at $Q^{2}=10 \mathrm{GeV}^{2}$. The solid circles are data for $N=Z$ nuclear matter from Ref. [18]. The solid curve is the calculation of $F_{2}^{A} / F_{2}^{D}$ for $N=Z$ nuclear matter from Cloët, Bentz, and Thomas [14, 15]. The dashed and dot-dashed curves are the ratios of quark distributions in a gold nucleus to those in a deuteron, for $u$ and $d$ quarks, respectively.

the deuteron. For an $N>Z$ nucleus (such as gold), the $\rho^{0}$ mean field leads to a stronger nuclear binding for the $u$ quarks compared to that for the $d$ quarks [14]. Hence, the medium modification of the $u$-quark distribution is enhanced, while the $d$-quark distribution is modified less by the medium, as illustrated in Fig. 1

The flavor dependence of the EMC effect is a promising experimental observable to distinguish among the plethora of models that can describe the EMC effect. In the simplest picture, we can consider the nuclear parton distribution functions (PDFs) to be just the nucleon PDFs smeared by the Fermi motion of the nucleons. In this picture it is natural that the nuclear modification would be very similar for $u$ and $d$ quarks. Similarly, the $Q^{2}$-rescaling model [19] also predicts no quark-flavor dependence for nuclear modifications. In contrast, the pion-excess model 20 22] would naturally predict flavordependent nuclear modifications arising from the different isospin composition of the pion cloud components of protons and neutrons.

Since the inclusive DIS experiments measure the combined nuclear modifications of $u$ and $d$ quarks, it is difficult to extract the quark-flavor dependence of the EMC effect in these experiments. However, it has been demonstrated [23] that the parity violating DIS asymmetry, $A_{P V}$, is very sensitive to the quark-flavor dependence of the EMC effect. Measuring $A_{P V}$ for a variety of $N \neq Z$ nuclei has the potential to provide a clean demonstration of flavor-dependent nuclear modification of the the quark distributions. Such a measurement is part of the large parity violating DIS program proposed [24] at the upgraded $12 \mathrm{GeV}$ JLab facility currently under construction.

Semi-inclusive DIS (SIDIS) on heavy nuclear targets, in which the flavor of the struck quark is tagged by the detected hadron, is also a promising experimental tool to search for the flavor-dependent EMC effect. Recently, Lu and Ma 25] pointed out that charged lepton SIDIS off nuclear targets and the deuteron can be used to probe the flavor content of the nuclear quark sea, which can help distinguish between the various models of the EMC effect. Indeed, a SIDIS experiment [26] aiming at a precise determination of flavor dependence of the EMC effect has also been proposed at the upgraded $12 \mathrm{GeV}$ JLab facility.

The focus of this Letter is pion-induced Drell-Yan processes, which are complementary to the DIS processes mentioned above and provide another experimental tool with which search for flavor-dependent effects in the nuclear modification of the nucleon structure functions. First, we will identify the experimental observables sensitive to the flavor-dependent EMC effect. Then we compare existing pion-induced Drell-Yan data with calculations using the CBT model, and examine how well the data can constrain the flavor dependence of the EMC effect. Finally, predictions for possible future pion-induced Drell-Yan experiments are presented.

The leading order Drell-Yan cross-section for a pion interacting with a nucleus is given by

$$
\begin{aligned}
& \frac{d \sigma_{\pi^{ \pm} A}^{2}}{d x_{\pi} d x_{2}}=\frac{4 \pi \alpha^{2}}{9 s x_{\pi} x_{2}} \\
& \quad \times \sum_{q} e_{q}^{2}\left[q_{\pi^{ \pm}}\left(x_{\pi}\right) \bar{q}_{A}\left(x_{2}\right)+\bar{q}_{\pi^{ \pm}}\left(x_{\pi}\right) q_{A}\left(x_{2}\right)\right],
\end{aligned}
$$

where $\alpha$ is the fine structure constant, $s$ is the center of mass energy squared, $x_{\pi}$ is the Bjorken scaling variable for the interacting quark in the pion and $x_{2}$ is the analogous quantity for the nucleon in the target nucleus. Quark flavor is labeled by $q$, where $e_{q}$ is the quark charge, the pion quark or antiquark distributions are labeled by $q_{\pi^{ \pm}}$and $\bar{q}_{\pi^{ \pm}}$, respectively, and the subscript $A$ indicates nuclear quark or antiquark distributions.

To explore the sensitivity of pion-induced Drell-Yan processes to a flavor-dependent EMC effect, we consider the three ratios $\frac{\sigma^{D Y}\left(\pi^{+}+A\right)}{\sigma^{D Y}\left(\pi^{-}+A\right)}, \frac{\sigma^{D Y}\left(\pi^{-}+A\right)}{\sigma^{D Y}\left(\pi^{-}+D\right)}$ and $\frac{\sigma^{D Y}\left(\pi^{-}+A\right)}{\sigma^{D Y}\left(\pi^{-}+H\right)}$, where $A$ represents a nuclear, $\mathrm{D}$ a deuteron and $\mathrm{H}$ a hydrogen target. Assuming isospin symmetry, which implies $u_{\pi^{+}}=d_{\pi^{-}}, \bar{u}_{\pi^{-}}=\bar{d}_{\pi^{+}}, \bar{u}_{\pi^{+}}=\bar{d}_{\pi^{-}}, u_{\pi^{-}}=d_{\pi^{+}}$and keeping only the dominant terms in each cross-section, one readily obtains

$$
\begin{gathered}
R_{ \pm}=\frac{\sigma^{D Y}\left(\pi^{+}+A\right)}{\sigma^{D Y}\left(\pi^{-}+A\right)} \approx \frac{d_{A}(x)}{4 u_{A}(x)}, \\
R_{A / D}^{-}=\frac{\sigma^{D Y}\left(\pi^{-}+A\right)}{\sigma^{D Y}\left(\pi^{-}+\mathrm{D}\right)} \approx \frac{u_{A}(x)}{u_{D}(x)}, \\
R_{A / H}^{-}=\frac{\sigma^{D Y}\left(\pi^{-}+A\right)}{\sigma^{D Y}\left(\pi^{-}+\mathrm{H}\right)} \approx \frac{u_{A}(x)}{u_{p}(x)} .
\end{gathered}
$$

The up and down nuclear quark distributions are labeled by $u_{A}$ and $d_{A}$ respectively, $u_{D}$ is the up quark distribution in the deuteron and $u_{p}$ the up quark distribution in the proton. Eqs. (2)-(4) demonstrate that these DrellYan cross-section ratios are very sensitive to the flavor 

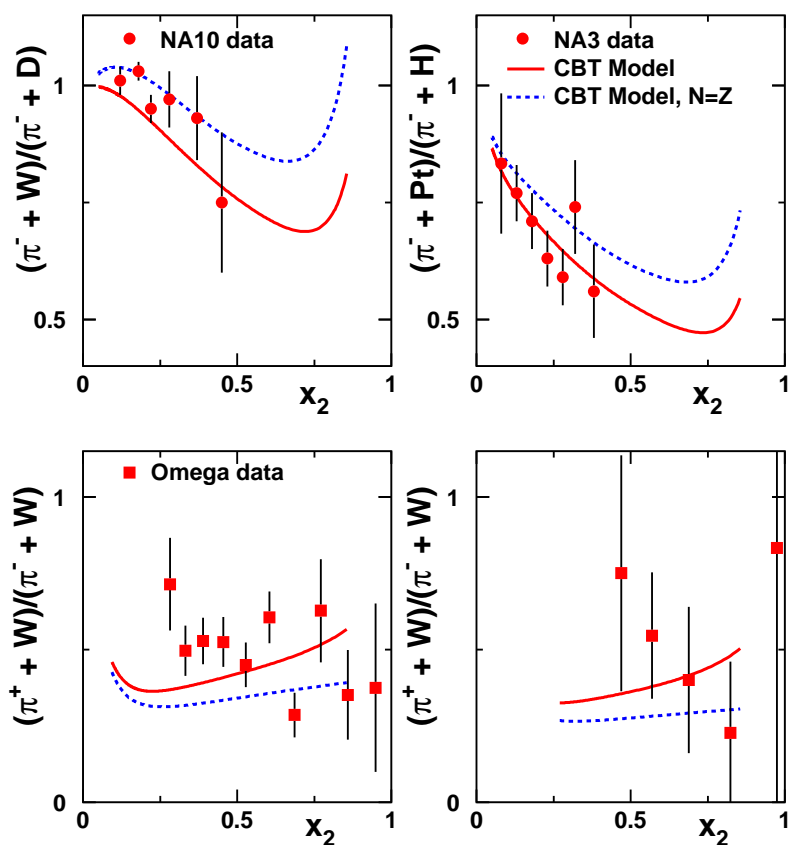

FIG. 2: The existing data for the ratios $\frac{\sigma^{D Y}\left(\pi^{-}+\mathrm{W}\right)}{\sigma^{D Y}\left(\pi^{-}+\mathrm{D}\right)}$ (upper left), $\frac{\sigma^{D Y}\left(\pi^{-}+\mathrm{Pt}\right)}{\sigma^{D Y}\left(\pi^{-}+\mathrm{H}\right)}$ (upper right) and $\frac{\sigma^{D Y}\left(\pi^{+}+\mathrm{W}\right)}{\sigma^{D Y}\left(\pi^{-}+\mathrm{W}\right)}$ (lower) versus the predictions using nuclear PDFs of the CBT model for tungsten (red solid) and $N=Z$ nuclear matter (blue dashed).

dependence of the EMC effect. Moreover, these ratios are not sensitive to the uncertainty of the pion structure functions, which are not yet determined accurately.

To study the sensitivity of the pion-induced Drell-Yan processes to the flavor dependence of the EMC effect we calculate these ratios using the nuclear and nucleon PDFs from the CBT model [14, 15]. Rather than using the approximate expressions of Eqs. (2)-(41), the expression for the Drell-Yan cross-section in Eq. (10) is used in the calculations. Terms involving heavy quarks $(s, c, b, t)$ are not included due to their negligible contributions. The pion-induced Drell-Yan cross-section for each nuclear target is determined using the nuclear PDFs from the CBT model. The free nucleon PDFs obtained from the CBT model are used to calculate the Drell-Yan cross-section from the deuteron, with $u_{D}=\left(u_{p}+d_{p}\right) / 2=d_{D}$. For the pion PDFs, we use the GRV-P LO parametrization [27]. As expected, the results are found to be insensitive to the choice of the pion PDFs.

In Fig. 2 we compare our calculations of the pioninduced Drell-Yan cross-section ratios with the existing data. The top left panel shows the ratio of $\frac{\sigma^{D Y}\left(\pi^{-}+\mathrm{W}\right)}{\sigma^{D Y}\left(\pi^{-}+\mathrm{D}\right)}$ from the NA10 experiment [7]. These plots contain both the $P_{\text {beam }}=286$ and $140 \mathrm{GeV}$ data sets, which are very similar. Our calculations are performed at $P_{\text {beam }}=$ $286 \mathrm{GeV}$, since most of the data was obtained at this energy. We use the PDFs of the CBT model [14, 15] at a fixed $Q^{2}$ of $25 \mathrm{GeV}^{2}$, which is approximately the mean $Q^{2}$ of the NA10 experiment. The top right panel shows the
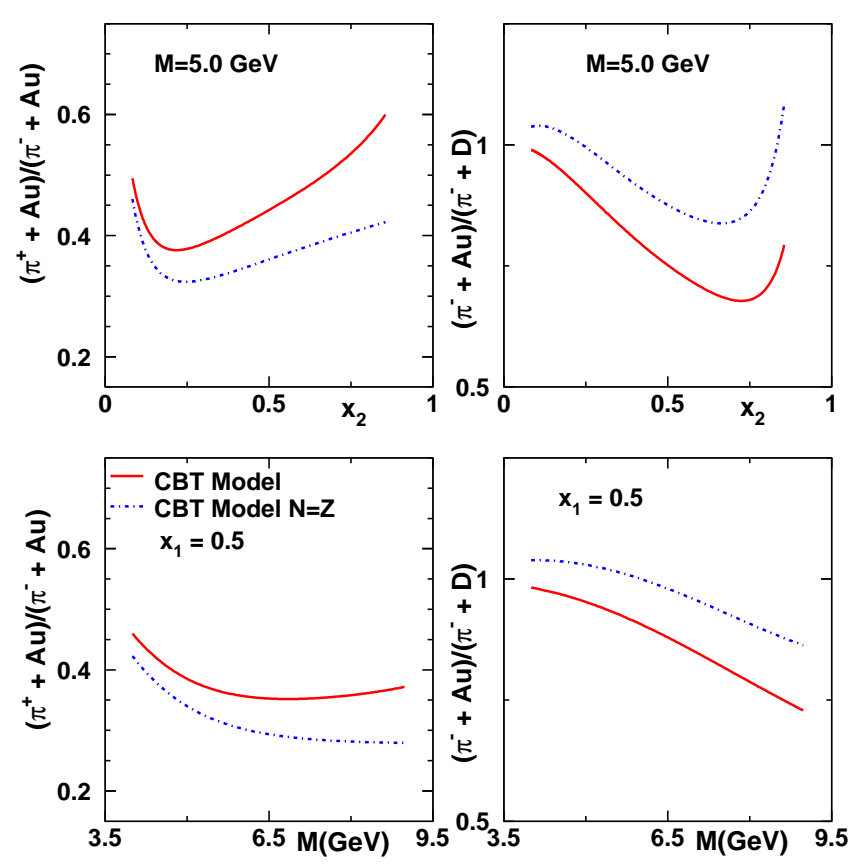

FIG. 3: The ratio of Drell-Yan cross sections for $\frac{\sigma^{D Y}\left(\pi^{+}+\mathrm{Au}\right)}{\sigma^{D Y}\left(\pi^{-}+\mathrm{Au}\right)}$ (upper left) and $\frac{\sigma^{D Y}\left(\pi^{-}+\mathrm{Au}\right)}{\sigma^{D Y}\left(\pi^{-}+\mathrm{D}\right)}$ (upper right) as a function of $x_{2}$, using nuclear PDFs from the CBT model for gold (red solid) and for $N=Z$ nuclear matter (blue dot-dashed). These calculations are performed for a pion beam energy of $160 \mathrm{GeV}$, at $Q^{2}=25 \mathrm{GeV}^{2}$. The lower panels show the same ratios but as a function of the dimuon mass, $M$, at a fixed $x_{1}=0.5$.

ratio $\frac{\sigma^{D Y}\left(\pi^{-}+\mathrm{Pt}\right)}{\sigma^{D Y}\left(\pi^{-}+\mathrm{H}\right)}$ from the NA3 experiment [28]. The data was collected using a $150 \mathrm{GeV} \pi^{-}$beam and the $Q^{2}$ range covered was $16.8 \leq Q^{2} \leq 70.6 \mathrm{GeV}^{2}$. Our calculations are performed for $P_{\text {beam }}=150 \mathrm{GeV}$ and $Q^{2}=25 \mathrm{GeV}^{2}$.

The solid curves in Fig. 2 are calculations using the flavor-dependent $u_{A}(x)$ and $d_{A}(x)$ from the CBT model with $N / Z=1.5$, corresponding approximately to the $N / Z$ values for the $\mathrm{Au}, \mathrm{W}$ and $\mathrm{Pt}$ nuclei. The dashed curves correspond to the calculated ratios using the nuclear PDFs from the CBT model for $N=Z$. Since $u_{A} / u_{D}=d_{A} / d_{D}$ in this case, the dashed curves are representative of the predictions for flavor-independent EMC models. Figure 2 shows that the NA10 data do not exhibit a clear preference for the flavor-dependent versus flavor-independent nuclear PDFs. In contrast, the NA3 data strongly favor the calculations using flavordependent nuclear PDFs.

The Drell-Yan ratio, $R_{ \pm}$, of Eq. (2) is an ideal experimental observable to search for flavor-dependent EMC effect, since it is directly proportional to $d_{A}(x) / u_{A}(x)$. The only existing data on $R_{ \pm}$is from the Omega collaboration [29]. The lower panels of Fig. 2 show the ratios of $\frac{\sigma^{D Y}\left(\pi^{+}+\mathrm{W}\right)}{\sigma^{D Y}\left(\pi^{-}+\mathrm{W}\right)}$ collected with $39.5 \mathrm{GeV}$ pions over a $Q^{2}$ range of $5.3 \leq Q^{2} \leq 7.3 \mathrm{GeV}^{2}$ and $16 \leq Q^{2} \leq 25 \mathrm{GeV}^{2}$. The calculations were performed at $Q^{2}=7 \mathrm{GeV}^{2}$ and 
$Q^{2}=20 \mathrm{GeV}^{2}$, respectively. Unfortunately, the existing data lack the precision for placing a useful constraint on the flavor dependence of the EMC effect.

The plan for the COMPASS collaboration [30] to measure Drell-Yan cross sections with $160 \mathrm{GeV}$ pion beams offers an exciting opportunity for a precise test of flavordependent EMC effect. Figure 3 shows the predictions of $R_{ \pm}$and $R_{A / D}^{-}$for the COMPASS kinematic coverage using the PDFs from the CBT model. The upper panels show the predictions as a function of $x_{2}$ for a fixed dimuon mass of $5 \mathrm{GeV}$, while the calculations for a fixed $x_{1}=0.5$ are shown in the lower panels as a function of dimuon mass. The significant difference between the predicted ratios using the flavor-dependent versus flavorindependent nuclear PDFs provide a strong motivation for such measurements in the future.

It is worth noting that several parametrizations of the nuclear parton distributions have been obtained from global analyses of DIS and Drell-Yan data [31 34]. However, pion-induced Drell-Yan data were not included in these global analyses. The sensitivities of the pioninduced Drell-Yan data to the flavor dependence of the nuclear PDFs, as shown in this study, strongly suggest that these data should be included in future global analyses.

In conclusion, we have examined the possibility of using pion-induced Drell-Yan processes as a sensitive experimental tool to study the flavor dependence of the modification of quark distributions in the nuclear medium. We suggest several Drell-Yan cross-section ratios as the most sensitive experimental quantities for such a study. The existing Drell-Yan data are not sufficiently accurate yet, although the NA3 data clearly favor the flavor-dependent over the flavor-independent nuclear PDFs. Precise future pion-induced Drell-Yan experiments can provide unique constraints that will help distinguish the various theoretical models and most importantly shed new light on the origins of the EMC effect.

This work was supported in part by the U.S. Department of Energy and the National Science Foundation.
[1] J. J. Aubert et al. (European Muon Collaboration), Phys. Lett. B 123, 275 (1983).

[2] J. Gomez et al., Phys. Rev. D 49, 4348 (1994).

[3] G. Bari et al., Phys. Lett. B 163, 282 (1985); A. C. Benvenuti et al., Phys. Lett. B 189, 483 (1987).

[4] A. M. Cooper et al., Phys. Lett. B 141, 133 (1984); H. Abramowicz et al., Z. Phys. C 25, 29 (1984).

[5] K. Ackerstaff et al. (HERMES Collaboration), Phys. Lett. B 475, 386 (2000) arXiv:hep-ex/9910071.

[6] J. Seely et al., Phys. Rev. Lett. 103, 202301 (2009) [arXiv:nucl-ex/0904.4448].

[7] P. Bordalo et al., Phys. Lett. B 193, 368 (1987).

[8] D. M. Alde et al., Phys. Rev. Lett. 64, 2479 (1990).

[9] P. L. McGaughey, J. M. Moss, and J. C. Peng, Annu. Rev. Nucl. Part. Sci. 49, 217 (1999).

[10] D. F. Geesaman, K. Saito and A. W. Thomas, Ann. Rev. Nucl. Part. Sci. 45, 337 (1995).

[11] P. R. Norton, Rept. Prog. Phys. 66, 1253 (2003).

[12] M. Ericson, A. W. Thomas, Phys. Lett. B148, 191 (1984).

[13] R. P. Bickerstaff, M. C. Birse, G. A. Miller, Phys. Rev. Lett. 53, 2532-2535 (1984).

[14] I. C. Cloët, W. Bentz and A. W. Thomas, Phys. Lett. B 642, 210 (2006) arXiv:nucl-th/0605061.

[15] I. C. Cloët, W. Bentz and A. W. Thomas, Phys. Rev. Lett. 102, 252301 (2009) [arXiv:nucl-th/0901.3559].

[16] W. Bentz, I. C. Cloet, J. T. Londergan and A. W. Thomas, arXiv:0908.3198 [nucl-th].

[17] G. P. Zeller et al. (NuTeV Collaboration) Phys. Rev. Lett. 88, 091802 (2002).

[18] I. Sick and D. Day, Phys. Lett. B 274, 16 (1992).
[19] F. E. Close, R. L. Jaffe, R. G. Roberts, and G. G. Ross, Phys. Rev. D 31, 1004 (1985).

[20] C. H. Llewellyn-Smith, Phys. Lett. B 128, 107 (1983).

[21] M. Ericson and A. W. Thomas, Phys. Lett. B 128, 112 (1983).

[22] E. L. Berger, F. Coester, and R. B. Wiringa, Phys. Rev. D 29, 398 (1984).

[23] I. C. Cloët, W. Bentz and A. W. Thomas, forthcoming publication.

[24] P. Souder et al., PR12-10-007, JLab Proposal to PAC-35, unpublished (2010).

[25] B. Lu and B.-Q. Ma, Phys. Rev. C 74, 055202 (2006).

[26] D. Dutta, D. Gaskell, K. Hafidi et al., PR12-09-004, JLab Proposal to PAC-34, unpublished (2009).

[27] M. Glück, E. Reya and A. Vogt, Z. Phys. C 53, 651 (1992).

[28] A. Michelini, CERN-EP/81-128 (1981); J. Badier et al., Phys. Lett. B 104, 335 (1981).

[29] M. Corden et al., Phys. Lett. B 96, 417 (1980).

[30] COMPASS Collaboratio, CERN Report No. CERN/SP SLC 96-14, SPSC/P 297.

[31] K. J. Eskola, V. J. Kolhinen, and C. A. Salgado, Eur. Phys. J. C 9, 61 (1999).

[32] K. J. Eskola, H. Paukkunen, and C. A. Salgado, JHEP 04, 065 (2009).

[33] M. Hirai, S. Kumano, and T.-H. Nagai, Phys. Rev. C 70, 044905 (2004).

[34] M. Hirai, S. Kumano, and T.-H. Nagai, Phys. Rev. C 76, 065207 (2007). 